\title{
Laryngeal Carcinoid Tumor
}

National Cancer Institute

\section{Source}

National Cancer Institute. Laryngeal Carcinoid Tumor. NCI Thesaurus. Code C6024.

A rare, well differentiated, low grade neuroendocrine neoplasm that arises from the larynx. 